\title{
Depth of anaesthesia predicts post-operative pain at six weeks following wide local breast tissue excision and sentinel node biopsy
}

\author{
Michael Wang ${ }^{1}$, Peter Beardsworth², Daniel Hensman ${ }^{3}$ \& Elizabeth Jonck ${ }^{4}$
}

(1) University of Leicester, UK; (2) Leicester Partnership NHS Trust; (3) University of Cambridge, UK; (4) University Hospitals of Leicester NHS Trust

\section{Background}

Some argue that light anaesthesia reduces post-operative mortality and morbidity (Monk, 2010). Others have argued that light anaesthesia may lead to post-operative psychological sequelae (Wang et al., 2004). Studies using the isolated forearm technique have demonstrated that intraoperative consciousness sufficient to enable response to command (with post-operative amnesia) is much more common than unplanned intraoperative awareness with post-operative recall (Russell, 1993). Does light anaesthesia with amnesia cause sensitisation of pain perception leading to elevated levels of post-operative pain?

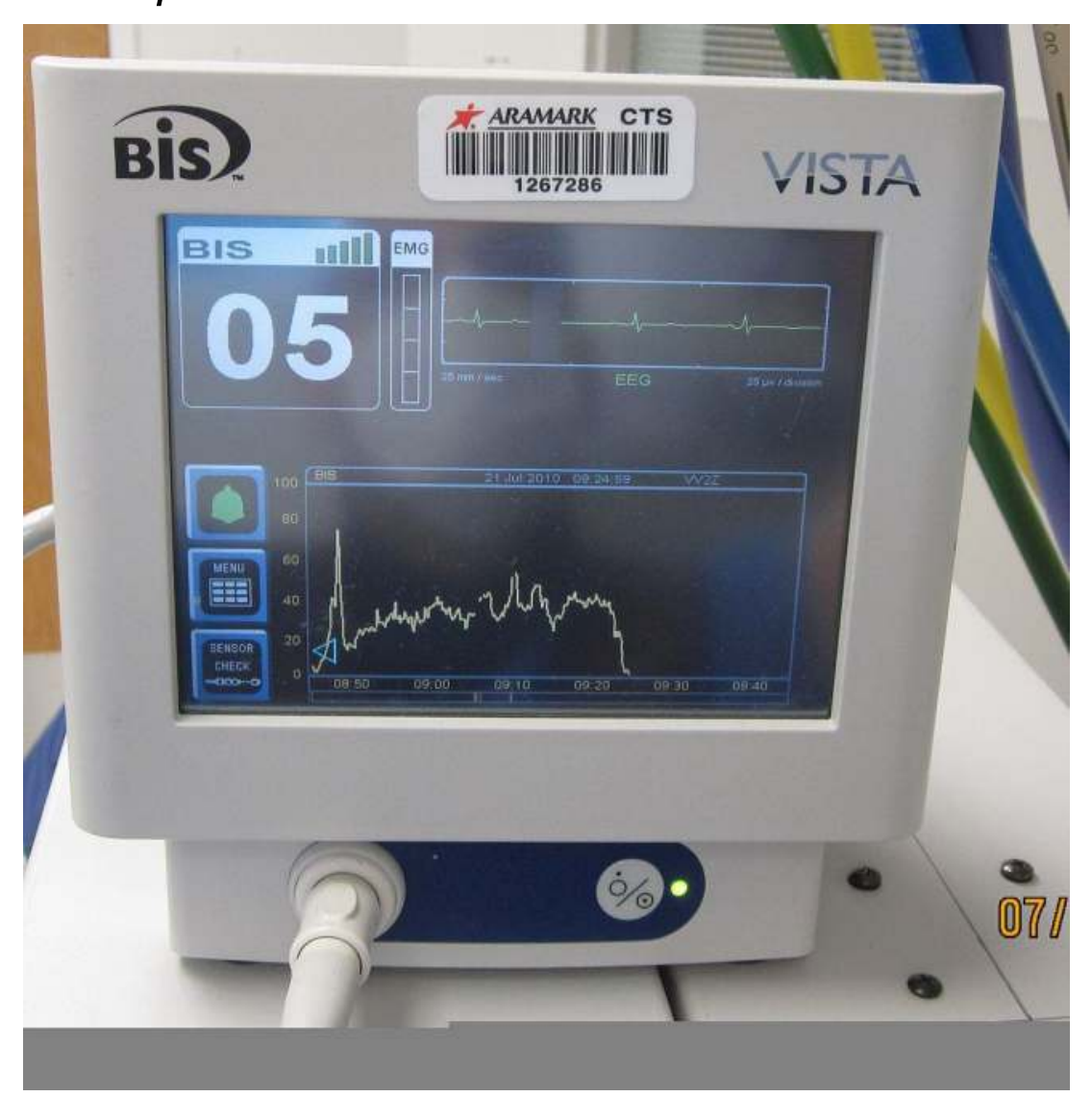

Material and methods

60 women (ASA 1 and 2) undergoing wide local excision of breast tissue and sentinel node biopsy recruited, mean age 62.1 range 31 to 85 . Anaesthetic technique: Propofol induction and Sevoflurane (1to1.5 MAC) in 50\% nitrous oxide maintenance along with Atracurium. Depth of anaesthesia monitored using Bispectral Index (Covidien Vista Monitor 185-0151 \& BISx 1850145-AMS)(MW,PB). The anaesthetist delivering the anaesthetic blind to BIS index. The anaesthetist was told the patient was "light" if the BIS index exceeded 55, and also told the patient was "deep" if the BIS index fell below 30. Average BIS recorded for each minute throughout.

\begin{tabular}{|l|l|l|l|}
\hline Pre-Op & $\mathbf{2 - 3}$ days & $\mathbf{6}$ weeks & $\mathbf{3}$ months \\
\hline STAI (state\&trait) & STAI (state) & STAI (state) & STAI (state) \\
\hline McGill Pain & McGill Pain & McGill Pain & McGill Pain \\
\hline VAS pain & VAS pain & VAS pain & VAS pain \\
\hline Pain Intensity & Pain Intensity & Pain Intensity & Pain Intensity \\
\hline Pain LoC & & & \\
\hline
\end{tabular}

Results and discussion

21/60 patients exceeded a BIS of 60 at some point during their surgery, with one as high as 88 . No patient had explicit postoperative recall. Significant positive correlations were found between (1) the BIS index post induction, (2) during the fiveminute epoch immediately following initial incision, and postoperative pain measures at six weeks $(r=0.43, p=0.002 ; r=0.285$, $p=0.039$ ), but not at the other two time points. The patient sample was divided using a BIS cut-off of 50 creating "deep" and "light" groups. An independent t-test demonstrated significant differences between these two groups on Present Pain Intensity $(t=2.42, p=0.019)$ and approached significance on the McGill Pain Questionnaire $(t=1.90, p=0.063)$ and on the visual analogue scale $(t=1.83, p=0.073)$ at six-week follow-up.

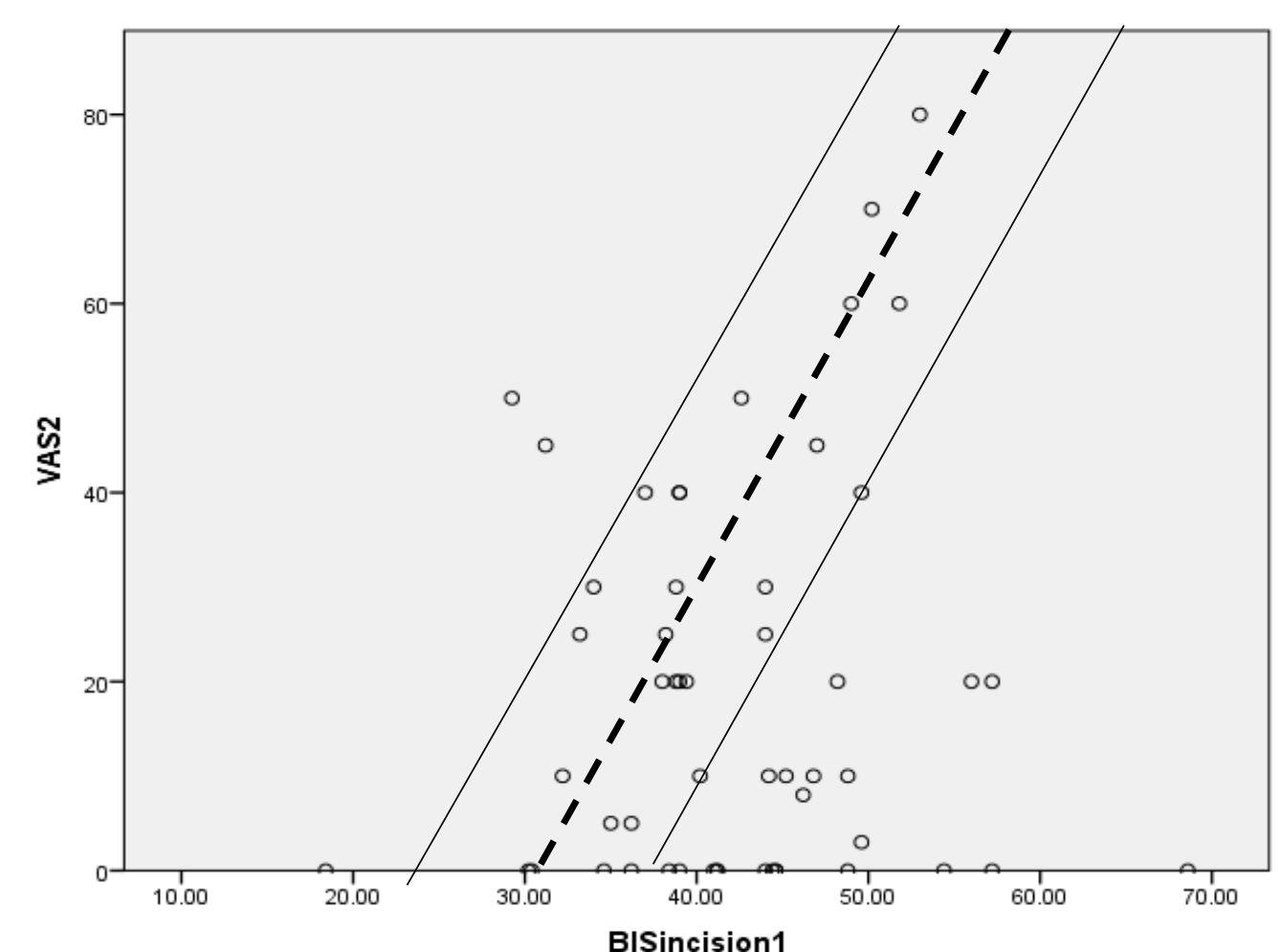

Scatterplot of VAS pain scores at 6 weeks against BIS during the 5-minute post incision epoch

\begin{tabular}{|c|c|c|c|}
\hline Variable & $\begin{array}{l}\text { Mean for } \\
\text { correlated } \\
\text { patients } \\
(\mathrm{N}=26)\end{array}$ & $\begin{array}{c}\text { Mean for } \\
\text { uncorrelated } \\
\text { patients } \\
(\mathrm{N}=23)\end{array}$ & $\begin{array}{c}\text { T-test } \\
\text { significance }\end{array}$ \\
\hline Age & 59.3 & 67.1 & 0.010 \\
\hline STAI state & 43.1 & 36.0 & 0.046 \\
\hline STAI trait & 36.1 & 31.0 & 0.026 \\
\hline $\begin{array}{c}\text { Overall mean } \\
\text { BIS }\end{array}$ & 40.0 & 43.9 & 0.008 \\
\hline MPQ pre-op & 4.4 & 1.5 & 0.014 \\
\hline
\end{tabular}

These results support the hypothesis that light anaesthesia may allow sensitisation to pain, specifically at 6 weeks postoperatively. How do we explain the absence of such a relationship at 2 days and 3 months? At 2 days, a variety of medical and surgical variables predominate in influencing pain perception, including analgesic dose, and so it is not surprising that more subtle psychological influences were masked. At 3 months most patients were pain-free and scoring minimally on pain measures (37/48), leaving little scope for significant correlation.

\section{Conclusion}

We found evidence of a positive relationship between intraoperative anaesthetic lightness and post-operative pain at six weeks following breast surgery in a cohort of 60 women. These findings, if replicated, suggest that routine depth of anaesthesia monitoring and responsive adjustment of anaesthetic dose may reduce post-operative pain. They also support the intriguing theory that intra-operative events and stimulation may be processed by the patient under light anaesthesia and have postoperative effects despite explicit amnesia.

\section{References}

Monk, T. Anesthetic depth is a predictor of mortality. Anesthesiology 2010; 112:1070-2

Wang, M, Russell, IF \& Logan, C. Light anaesthesia without explicit recall during hysterectomy is associated with increased post-operative anxiety over a 3-month follow-up period. British Journal of Anaesthesia 2004; 93: 492-493

Russell, I.F. Midazolam-alfentanyl: an anaesthetic? An investigation using the isolated forearm technique. British Journal of Anaesthesia 1993; 70: 42-46.

\section{University Hospitals of Leicester Wits Leicestershire Partnership WHS NHS Trust

